\title{
La lexicografía en los planes de estudio de traducción e interpretación en España
}

\author{
(Lexicography curriculum in Translation and \\ Interpretation in Spain)
}

Jesús CAMACHO NiÑO Universidad de Jaén

Fecha de recepción: 18 de junio de 2013

Fecha de aceptación: 15 de julio de 2013

Resumen: Es un hecho innegable que el diccionario es una herramienta de gran ayuda para el trabajo de traducción e interpretación. De otro lado, tampoco podemos obviar la gran complejidad que actualmente presentan los diccionarios. Esta intrincada red de informaciones lingüísticas, impone un conocimiento de los principios que rigen la construcción y el uso del diccionario. Por ello, para obtener el máximo rendimiento de estas obras, es necesario tener una formación específica en la disciplina lexicográfica.

Con estas premisas, a lo largo de las siguientes páginas analizaremos la situación de los estudios lexicográficos dentro de los planes de estudio universitarios de Traducción e interpretación.

El análisis de las distintas universidades españolas que ofertan esta formación superior nos muestra que el principal interés que presenta la lexicografía en la formación de traductores se halla en la lexicografía especializada. Es decir, en las asignaturas de terminología.

Si bien la principal representación de la lexicografía la encontramos en los módulos de terminología, también podemos hallar presencia de esta en otras asignaturas, aunque de manera mucho más reducida. Nos referimos a materias como lengua española o lingüística - entre otras-, las cuales también han sido revisadas en nuestro estudio.

Palabras clave: Lexicografía, Traducción e interpretación, Universidad, Terminología, Lengua española y lingüística.

Abstract: Needless to say, the dictionary is a useful tool in Translation and Interpreting. In addition, it is conceived of as a complex network of linguistic information, which serves to establish the overriding principles for the 
structure and use of it. Owing to this, having a specific training in the field of Lexicography is required to gain profit from the dictionary. A survey of different Spanish Universities that offer Translation and Interpreting shows the branch of Specialized Lexicography is essential in the translator's training agenda. Likewise, subjects such as Terminology mainly, Spanish and Linguistics among others are covered from a lexicographical approach.

The aim of this paper is to analyze the role of Lexicography in the curricula of Translation and Interpreting.

Key words: Lexicography, Translation and Interpretation, University, Terminology, Spanish language and linguistic

\section{INTRODUCCIÓN}

El propósito de este trabajo es el de analizar el lugar que ocupa la disciplina lexicográfica en los actuales planes de estudio de traducción e interpretación de las universidades públicas de España.

El punto de partida se encuentra en la reflexión sobre la importancia del diccionario en el trabajo traductológico. Efectivamente, todos somos conscientes de la cantidad de información que pueden obtener los profesionales de la traducción y la interpretación de este producto lingüístico. El artículo lexicográfico es la unidad funcional que da su aspecto al diccionario. Su morfología ha sido diseñada para ofrecer de manera condensada, gran cantidad de información lingüística sobre la codificación y decodificación de una lengua. Podríamos decir que en ellos se hace una disección lingüística, si se permite el símil, de las voces que forman una lengua. No podemos negar que una herramienta de estas características es el complemento perfecto para la labor del traductor.

$\mathrm{Si}$ es un hecho innegable la necesidad que el traductor tiene del diccionario, no lo es menos el continuo desarrollo experimentado por la lexicografía a lo largo de los siglos, especialmente desde la segunda mitad del XX con el nacimiento de la Metalexicografía. Avance que ha hecho del diccionario un complejo entramado de información lingüística. Conocer los aspectos definitorios del diccionario (el tipo de léxico contenido, la organización macroestructural, la lematización, la ordenación de las acepciones o la tipología definicional, entre otros) facilitará tanto la selección del más adecuado a las necesidades del usuario, como la correcta interpretación y valoración de la información contenida en las columnas del mismo.

Es la complejidad del diccionario que intentamos hacer patente arriba, la que nos lleva a defender la siguiente postura. El máximo 
aprovechamiento de su potencial, pasa por conocer las características que configuran y dan forma a las obras lexicográficas, a la vez que tener un conocimiento específico de los principios por los que se rigen.

Ante esto, es totalmente deseable y necesario que un profesional del uso del diccionario, como es el traductor y el intérprete, tenga formación en este campo de estudio.

Por ello, a lo largo de las siguientes páginas analizaremos, en la medida que nos lo permita el espacio del que disponemos, cómo ha sido la recepción de los estudios lexicográficos en los actuales planes docentes de las universidades públicas españolas correspondientes al grado en Traducción e interpretación.

\section{LOS ESTUDIOS UNIVERSITARIOS DE TRADUCCIÓN E INTERPRETACIÓN EN ESPAÑA} Y LA LEXICOGRAFÍA

Los primeros centros de enseñanza de la traducción en España datan de los años setenta del pasado siglo. Concretamente, en el año 1974 se fundó en el primero de ellos, el Instituto Universitario de Lenguas Modernas y Traductores, adscrito a la Universidad complutense de Madrid. Sus principales intereses se centraban en la traducción literaria. Otros centros que surgieron a partir de esta fecha fueron los de Granada, autónoma de Barcelona y Las Palmas de Gran Canaria. Sin embargo, la plena integración de estos estudios en la oferta académica de las universidades españolas no se produjo hasta la década de los noventa con la aparición de la Licenciatura en Traducción e interpretación (cf. Vega, 2004: 568-69).

Actualmente, y tras muchos cambios y ajustes en la regulación de los estudios universitarios, esta licenciatura se ha convertido en un grado que se imparte en dieciséis universidades públicas españolas. A saber:

Universidad autónoma de Barcelona

Universidad complutense de Madrid

Universidad de Alicante

Universidad de Córdoba

Universidad de Granada

Universidad de las Palmas de Gran Canaria

Universidad de Málaga

Universidad de Murcia

\author{
Universidad de Salamanca \\ Universidad de Valencia \\ Universidad de Valladolid \\ Universidad de Vigo \\ Universidad del País Vasco \\ Universidad Jaume I \\ Universidad de Pablo de Olavide \\ Universidad Pompeu Fabra
}

Como podemos ver, los estudios universitarios sobre esta disciplina tienen una notable presencia en las universidades públicas españolas. Junto a estos, existen centros de carácter privado que incluyen esta titulación dentro de su oferta académica. Pero, por cuestiones de espacio y 
sin desmerecer el trabajo realizado en ellos, hemos decidido centrar nuestro esfuerzo e interés en las universidades públicas.

La metodología que hemos seguido para elaborar este trabajo se basa en la consulta y comparación de los temarios de las universidades que incluyen estos estudios en su oferta docente. El fin es obtener una visión clara del lugar que ocupa la lexicografía dentro de estos estudios. El resultado de la comparación nos ayudará a evaluar de una manera objetiva, en la medida de lo posible, la situación en la que se encuentra la lexicografía dentro de la traducción; facilitando la emisión de propuestas de mejora.

El rastreo del temario lexicográfico en los distinto planes de estudio ha sido lo más exhaustivo posible. Gracias a esto, en la mayoría de los casos hemos obtenido los datos necesarios. Las búsquedas realizadas en las universidades anteriormente comentadas han sido fructíferas en catorce ocasiones, no siendo así en dos.

Nos referimos a la Universidad autónoma de Barcelona y a la Universidad de Valladolid. En el primer caso, no hemos podido obtener ningún tipo de información sobre las asignaturas y los temarios impartidos en la titulación. La Universidad de Valladolid sí integra información del temario de sus asignaturas pero no en todas. Esto nos ha impedido confirmar la presencia de los estudios lexicográficos en el grado de Traducción e interpretación impartido en este centro.

Por tanto, aunque son dieciséis las universidades públicas que imparten esta titulación, por la imposibilidad de acceder a las guías académicas de dos de ellas, analizaremos un total de catorce planes de estudio.

2. La LEXICOGRAFía EN LOS PLANES dE ESTUdio DE TRADUCCIÓN E INTERPRETACIÓN

Es un hecho confirmado tras nuestro análisis, que el desarrollo y uso del diccionario son una materia de interés para los grados de traducción e interpretación de las universidades públicas de España.

Otra cuestión es el lugar donde se integran estos conocimientos. Lo más habitual, ocurre en todos los casos, es que el temario sobre la lexicografía se incluya en las asignaturas de Terminología. Sin embargo, también encontramos estos contenidos, aunque en menor medida, en otras. A saber: Lengua, Lingüística aplicada a la traducción, Documentación para traductores e intérpretes e Informática.

Como vemos, los contenidos lexicográficos no se imparten todos en una misma materia. Si bien, también encontramos una excepción a esta 
afirmación. Una asignatura dedicada exclusivamente a la lexicografía dentro de la traducción. Esta es Recursos lexicográficos aplicados a la traducción, de la Universidad de Salamanca.

También nos ayudará en nuestras reflexiones sobre la importancia de la lexicografía en estos estudios conocer el tipo de asignatura en la que se incluyen. Si observamos la tipología de aquellas que son de nuestro interés, las que integran a la lexicografía son del tipo básico u obligatorio. Módulos que los alumnos están obligados a cursar y superar para completar su formación como traductor e intérprete. De esto, podemos deducir que el uso de los diccionarios es considerado un conocimiento totalmente necesario para el profesional de la traducción la interpretación.

Sin embargo, aunque esté presente en la totalidad de los centros públicos, el volumen dedicado a ella no es el mismo en todos. Hay universidades en las que hay más presencia que en otras. Este es el caso de la Universidad de Córdoba y Salamanca. Ambas tienen un total de cuatro asignaturas donde se imparte lexicografía. La primera de ellas lo hace en Lengua española I, Herramientas profesionales para la traducción, Terminología e informática aplicada a la traducción. La segunda reserva para la instrucción diccionarística Recursos lexicográficos para la traducción, Documentación para la traducción, Terminología aplicada a la traducción y Gestión terminológica y de proyectos.

En el lado opuesto, también contamos con algún ejemplo ilustrador. El centro con menos asignaturas de temario lexicográfico es la Universidad Pablo de Olavide en Sevilla. Esta solo cuenta con un módulo para el estudio del diccionario (Terminología). El resto de universidades se encuentran a medio camino entre estos polos expuestos, fluctuando entre dos y tres asignaturas.

Volviendo a la ubicación de la lexicografía, no es sorpresa el lugar que ocupa. Hay una relación indivisible entre traductor, diccionario y terminología que intentaremos explicar.

Como ya sabemos, el diccionario es una herramienta imprescindible para la realización del trabajo traductológico. A esto añadimos que un área profesional con mucho potencial es la traducción especializada, lo que impone un profundo conocimiento del funcionamiento de las lenguas de especialidad. Finalmente, por su parte, la lexicografía ha mostrado prácticamente desde sus estadios más primitivos, un gran interés por el léxico terminológico. Tal es este, que el tiempo ha dado lugar al establecimiento de una rama específica de la lexicografía para el estudio y elaboración de diccionarios especializados, la lexicografía especializada o de especialidad. 
La confluencia de las disciplinas terminológica y lexicográfica se materializa en el diccionario especializado, fundamental para la realización de traducciones especializadas. Esto prueba la necesidad del traductor de poseer conocimientos terminológicos y lexicográficos. De ahí que sea justificada la presencia de la lexicografía en las asignaturas de terminología.

\subsection{Terminología}

Los estudios diccionarísticos presentes en los grados de traducción han sido diseñados en función de las necesidades de los futuros trabajadores de esta rama. Si la traducción especializada es un ámbito con grandes posibilidades profesionales para este colectivo y para desempeñarla es necesario el uso de un diccionario diatécnico, es normal que los temarios vuelquen su interés en los aspectos lexicográficos que traten esto. Con la docencia de las asignaturas de terminología se busca que el discente sea autónomo en la búsqueda y utilización de fuentes y recursos de ambas disciplinas con el fin de realizar trabajos traductológicos sobre las lenguas de especialidad.

La comparación de los distintos temarios evidencia una acusada homogeneidad en ellos. La Terminografía es una subdisciplina presente en todas las asignaturas de Terminología. Esta se puede definir como "parte de la lexicografía que estudia la actividad lexicográfica de la terminología (Sin.: lexicografía terminológica)" (DLP, 1995: s.v. terminografía $)^{1}$. Es decir, la investigación y elaboración de productos lexicográficos especializados. Este es el eje central de los estudios lexicográfico en los estudios de traducción.

Dentro de la terminografía, los contenidos impartidos son principalmente metodológicos. Orientados a mostrar el funcionamiento interno de estas obras para conocerlas y manejarlas óptimamente.

Los aspectos más comunes impartidos sobre la subdisciplina en cuestión son los siguientes:

- Principios teóricos de la terminografía,

- Documentación, identificación y resolución de problemas terminológicos y terminográficos,

- Selección de términos,

- Periodización del trabajo terminográfico,

- Elaboración de fichas terminológicas

- Tipología de los productos terminográficos: bases de datos terminológicas, corpus y glosarios.

${ }^{1}$ DLP: Diccionario de lexicografía práctica. 
En menor medida, en esta asignatura también se incluye, en ocasiones, enseñanzas sobre Terminótica: "(cruce entre terminología y terminografía) técnica para el tratamiento informático de la terminología" (DLP, 1995: s. v. terminótica). Esto no ocurre en todas las universidades. Los centros que incluyen la terminótica en la asignatura de Terminología son las universidades de Córdoba, Las Palmas de Gran Canaria y Salamanca.

En cuanto a los contenidos impartidos sobre estas cuestiones, los más repetidos son aquellos relacionados con las posibilidades de aplicación de la informática al trabajo terminológico. Concretando más, hablamos de aplicaciones informáticas sobre la selección y extracción de términos, elaboración y evaluación de corpus lingüísticos o industrias de la lengua.

Un caso excepcional dentro de este conjunto de asignaturas es el de la Universidad de Valencia. Terminología y lexicografía se centra exclusivamente en aspectos que podríamos entroncar en la lexicografía especializada. Su temario está dividido en tres bloques. El primero trata los aspectos comunes y divergentes de ambas disciplinas. La segunda queda reservada para los productos lexicográficos (tipos de diccionarios, enciclopedias, tesauros, etc.). Y finalmente, la tercera recoge los fundamentos, metodología y aplicaciones de la terminografía.

La observación y el estudio de los programas a nuestra disposición confirman la importante presencia de la lexicografía en la asignatura de terminología impartida en las carreras de Traducción e interpretación. Tras el trabajo realizado con nuestras fuentes de información, podemos decir que los conceptos lexicográficos que predominan son los relacionados con la metodología terminográfica.

\subsection{Lengua}

Como decíamos anteriormente, las asignaturas de Terminología no son las únicas que tratan la lexicografía. Hay otras en las que también se da cabida a estas cuestiones. Si bien, la amplitud con la que son tratadas no es la misma. Las asignaturas de Lengua son otra de las ubicaciones que algunas universidades han elegido para la lexicografía. Los centros universitarios que se han decantado por esta opción son los de Córdoba, Granada, Jaume I, Madrid (complutense), Málaga y Valencia.

Si los contenidos lexicográficos de las asignaturas de Terminología eran aquellos concernientes a la terminografía, los de las asignaturas de Lengua son sensiblemente distintos. Estos se centran principalmente en los conceptos básicos de lexicografía y en la tipología de diccionarios. El fin de 
estos estudios no es otro que forma al alumno para que pueda elegir y manejar de manera eficaz los distintos tipos de diccionarios ${ }^{2}$

\subsection{Lingüística aplicada a la traducción}

Dos son las universidades que dentro de esta asignatura introducen materia lexicográfica. La Universidad de Alicante y la Universidad de Granada. Los contenidos que nos interesan son impartidos en los bloques correspondientes al significado y su codificación ${ }^{3}$.

\subsection{Documentación para traductores e intérpretes}

En este caso, tenemos cuatro centros: Madrid (complutense), Las Palmas de Gran Canaria, Salamanca y País Vasco.

El objetivo fundamental que buscan estas asignaturas es instruir al alumno en los métodos de búsqueda de información. Tras cursar estos estudios, el futuro traductor debe conocer los recursos que tiene a su disposición para reunir información general y especializada que le sea de ayuda en su trabajo traductológico ${ }^{4}$. Por ello, el diccionario como fuente de información lingüística y cultural se convierte en uno de los objetos de estudio.

\subsection{Informática} Valencia.

Este caso podemos ilustrarlo con tres ejemplos: Córdoba, Murcia y

Según nos dice la guía académica de la Universidad de Valencia "esta asignatura está concebida para desarrollar contenidos instrumentales referidos al dominio de las tecnologías de la información y la comunicación y de las técnicas de traducción asistida y localización" ${ }^{5}$. Por tanto, los esfuerzos de los docentes se centrarán principalmente, en la instrucción sobre los diccionarios electrónicos. Deberán ilustrar a alumno en las ventajas y desventajas que presentan estos frente a los diccionarios en papel, así como las diversas posibilidades que se abren con su uso.

\footnotetext{
${ }^{2}$ Así lo especifican las distintas guías docentes en el apartado de competencias. Disponible online en los enlaces recogidos en la sección de Referencias bibliográficas.

${ }^{3}$ Confróntense las guías docentes de las asignaturas.

${ }^{4}$ Confróntese el apartado de objetivos de la guía correspondiente a la Universidad de Salamanca.

${ }^{5}$ Disponible online. Consúltese la sección de Referencias bibliográficas.
} 


\subsection{Recursos lexicográficos para la traducción}

Hasta el momento, ya hemos visto las asignaturas donde habitualmente se integran los estudios lexicográficos. Sin embargo, no querríamos concluir esta investigación sin dedicar unas líneas a otro módulo. Este es el único concebido y orientado únicamente hacia la teoría lexicográfica. Como ya veíamos anteriormente, la lexicografía no poseía un espacio propio dentro de las carreras de Traducción, sino que se insertaba dentro de otras asignaturas.

Por el contrario, todos los créditos de Recursos lexicográficos para la traducción se han reservado para la enseñanza de esta disciplina. Los objetivos de la asignatura, tal y como reza su guía académica son "familiarizar de forma práctica al estudiante con los conocimientos necesarios para el manejo de los recursos en soporte papel y digital para el desarrollo de la teoría y la práctica la lexicografía en el futuro traductor e intérprete" ${ }^{\prime \prime}$. En esta se busca que el alumno conozca las características y tipos de diccionarios existentes. De esta manera tendrá la formación suficiente para elegir aquel que más se ajuste a sus necesidades. $Y$ a la vez, le permitirá evaluar objetivamente la información que le proporciona la obra lexicográfica.

Para ello, se ha diseñado un temario que se articula en tres ejes: morfología (macroestructura y microestructura), tipología (papel, electrónicos, bilingües y especializados) y evaluación crítica (análisis crítico de los diccionarios) ${ }^{7}$.

\section{CONCLUSIONES}

El estudio de las características y principios que rigen el funcionamiento y manejo de los diccionarios es una materia que los alumnos de los grados en Traducción e interpretación deben superar obligatoriamente. Por tanto, podemos inferir que son conocimientos muy necesarios para estos profesionales.

Tal y como decíamos anteriormente ${ }^{8}$, una de las características del estudio de la lexicografía en estas carreras es que los temarios de esta no ocupan un lugar propio, sino que se integran en otras asignaturas. Principalmente, estas son: Terminología, Lengua, Lingüística aplicada a la traducción, Documentación para traductores e intérpretes e Informática.

\footnotetext{
${ }^{6}$ Disponible en la sección de Referencias bibliográficas.

${ }^{7}$ Confróntese el apartado de contenidos de la guía docente de la asignatura. Disponible en la sección de Referencias bibliográficas

${ }^{8}$ Véase apart. 3: La lexicografía en los planes de estudio de Traducción e Interpretación.
} 
De estas, la que más peso tiene es la primera de ellas, Terminología. Todas las universidades que han sido objeto de nuestro estudio incluyen la mayoría de su temario lexicográfico en esta. La razón radica en la importancia que tienen para este colectivo la traducción científico-técnica y las grandes posibilidades que ofrece el uso del diccionario en este ámbito. En cuanto a las otras asignaturas, la dispersión del conocimiento lexicográfico no es tan homogénea como en el caso de la Terminología, pero la utilidad de sus contenidos es innegable.

La inclusión de esta disciplina a los estudios traductológicos está enfocada desde un punto de vista pragmático. Con esto queremos decir que frente a la gran variedad de conceptos y contenidos de la lexicografía, se han seleccionado aquellos que le son de más utilidad al traductor y se han integrado en las materias más afines a estos.

Uno de los aspectos que nos ha llamado la atención de la enseñanza de la lexicografía en los estudios traductológicos ha sido la falta de atención que se le presta a la práctica lexicográfica, punto desde el que se origina todo diccionario. En el párrafo anterior dijimos que la orientación de la lexicografía en estos estudios era eminentemente pragmática. Sin embargo, la reflexión teórica nacida de esta práctica puede ser uno de los mejores sustratos sobre los que construir un diccionario. Aunque la elaboración de diccionarios no sea el objeto central del trabajo del traductor, sus aportaciones pueden ser fundamentales en esta tarea. Muchos de los diccionarios bilingües han nacido de la práctica de la traducción.

Por otra parte, tampoco podemos olvidar la importancia que cobra en este ámbito la intervención de la informática y las nuevas tecnologías. Las posibilidades que actualmente nos ofrece el mundo de los ordenadores le ha dado un giro completo a la elaboración de diccionarios. Hoy en día, podemos encontrar software orientados al tratamiento y procesamiento de los textos que formarán parte de las fuentes primarios del diccionario. O la existencia en formato digital de los grandes corpus lingüísticos, tanto de las lenguas especializadas como de la general, es otra herramienta informática con grandes posibilidades para aquellos dedicados a la creación de diccionarios.

Por lo dicho, creemos que sería muy provechoso incluir estas cuestiones en los temarios de las asignaturas que recogen la enseñanza de la lexicografía en las carreras de traducción. Y no es otro afán el que nos mueve a defender esto, que pensar en los beneficios que se aportarían a estas dos disciplinas. Los traductores contarían con mejores recursos lexicográficos para realizar su trabajo, a la vez que los lexicógrafos se 
beneficiarían de las observaciones hechas por los traductores sobre los diccionarios.

Para finalizar solo queríamos corroborar que el uso del diccionario es fundamental para la correcta realización del trabajo traductológico. Prueba de ello es que en todas las universidades públicas españolas en las que se estudia el grado en Traducción e interpretación se imparten clases de esta disciplina lingüística

\section{REFERENCIAS BIBLIOGRÁFICAS}

Fuentes primarias

UNIVERSIDAD AUTÓNOMA DE BARCELONA. GRADO EN TRADUCCIÓN E INTERPRETACIÓN. Disponible online:

http://www.uab.es/servlet/Satellite/estudiar/todos-los-

estudios/informacion-general/traduccion-e-interpretacion-grado-eees-

1099409747826. html ?param1 $=1228291018508 \&$ param10 $=3$

[Consultado el 20 de julio de 2012].

UnIVERSIDAD COMPLUTENSE DE MADRID. GRADO EN TRADUCCIÓN E INTERPRETACIÓN. Disponible online: http://tei.cesfelipesegundo.com/ [Consultado el 20 de julio de 2012].

Universidad dE ALICANTE. GRADO EN TRADUCCIÓN E INTERPRETACIÓN. Disponible online: http://dti.ua.es/es/presentacion/presentacion.html [Consultado el 20 de julio de 2012].

Universidad de CóRdoba. GRAdo eN TRADUCCIÓN E INTERPRETACIÓN. Disponible online:

http://www.uco.es/filosofiayletras/gtradinterpretacion/index.html [Consultado el 20 de julio de 2012]

Universidad DE GRANADA. GRADO EN TRADUCCIÓN E INTERPRETACIÓN. Disponible online: http://grados.ugr.es/traduccion/ [Consultado el 20 de julio de 2012].

Universidad de Las Palmas de Gran Canaria. Grado en Traducción e INTERPRETACIÓN. Disponible online:

http://www.ulpgc.es//index.php?pagina=estudios\&ver=weees002\&tipo plan=\&codigo=141_4003_40_00 [Consultado el 20 de julio de 2012].

UNIVERSIDAD DE MÁlAGA. GRADO EN TRADUCCIÓN E INTERPRETACIÓN. Disponible online: http://webdeptos.uma.es/traduccion/ [Consultado el 20 de julio de 2012].

Universidad DE MURCIA. GRAdo en TRADUCCIÓN E INTERPRETACIÓN. Disponible online:

http://www.um.es/web/letras/contenido/estudios/grados/traduccioningles [Consultado el 20 de julio de 2012]. 
Universidad de Salamanca. Grado en Traducción E INTERPRETACIÓN. Disponible online: http://www.usal.es/webusal/node/4622 [Consultado el 20 de julio de 2012].

Universidad DE VALENCIA. GRAdo EN TRADUCCIÓN E INTERPRETACIÓN: Disponible online:

http://www.uv.es/uvweb/filologia-traduccion-comunicacion/es/estudiosgrado/grados/grau-1285848125087/Titulacio.html id =1285847388019 [Consultado el 20 de julio de 2012].

UnIVERSIDAD DE VALLADOLID. GRADO EN TRADUCCIÓN E INTERPRETACIÓN. Disponible online:

http://www.uva.es/opencms/consultas/planesestudios/asignaturas?an o_academico $=1213 \&$ codigo_plan $=423$ [Consultado el 20 de julio de 2012].

Universidad de Vigo. GRAdo En TRAducción E INTERPRETACIÓN. Disponible online:

http://webs.uvigo.es/victce/index.php?option=com_content\&task=view \&id $=1111$ \&ltemid $=352 \&$ lang $=$ gl [Consultado el 20 de julio de 2012].

UnIVERSIDAD dEL PAís VASCO. GRADO EN TRADUCCIÓN E INTERPRETACIÓN. Disponible online:

http://www.ehu.es/p200-

content/es/pls/entrada/plew0040.htm_siguiente?p_sesion=\&p_cod_idi oma $=C A S \& p \_e n \_p o r t a l=S \& p \_a n y o A c a d=a c t \& p \_c o d \_c e n t r o=130 \& p$ cod_plan=GTRADU10\&p_menu=intro [Consultado el 20 de julio de 2012].

Universidad JaUme I. Grado en TRADUCCIÓn E INTERPRETACIÓN. Disponible online:http://www.uji.es/infoest/estudis/grau/esp/ah/trad.html [Consultado el 20 de julio de 2012].

Universidad Pablo de Olavide. Grado en Traducción e INTERPREtación. Disponible online:

http://www.upo.es/fhum/contenido?pag=/portal/fhum/oferta_academic a/Guias_Docentes/Guias_Docentes_2011_2012/Guia_TEI_Ingles_20 11_12\&menuid [Consultado el 1 de agosto de 2012].

Universidad POMPEU Fabra. Grado en TRADUCCIÓN E INTERPRETACIÓN. Disponible online:

http://www.upf.edu/factii/es/factii_grau/presentacio/index.html [Consultado el 1 de agosto de 2012]. 
Otras fuentes

MARTínEZ DE SOUSA, J., Diccionario de lexicografía práctica, Barcelona: Vox, 1995.

VEGA, M. Á., "De la Guerra Civil al pasado inmediato". En Lafarga, F. y L. Pegenaute (eds.): Historia de la traducción en España, Salamanca: Ambos Mundos, 2004.

ANEXO

SIGLAS DE LAS UNIVERSIDADES

$\mathrm{UAB}=$ Universidad autónoma de Barcelona

$\mathrm{UCM}=$ Universidad complutense de Madrid

$\mathrm{UA}=$ Universidad de Alicante

$U C=$ Universidad de Córdoba

UGR $=$ Universidad de Granada

ULPGC $=$ Universidad de Las Palmas de Gran

Canaria

UMA. $=$ Universidad de Málaga

$U M=$ Universidad de Murcia

USAL $=$ Universidad de Salamanca

UVAL $=$ Universidad de Valencia

$U V A=$ Universidad de Valladolid

$U V I=$ Universidad de Vigo

UPV $=$ Universidad del País Vasco

$U \mathrm{UI}=$ Universidad Jaume I

UPDO $=$ Universidad Pablo de Olavide

UPF $=$ Universidad Pompeu Fabra 
ASIGNATURAS POR UNIVERSIDAD

\begin{tabular}{|c|c|c|c|}
\hline \multicolumn{4}{|c|}{ UNIVERSIDAD COMPLUTENSE DE MADRID } \\
\hline Asignatura & Curso & Carácter & Carga lectiva \\
\hline Lengua $A$ & 10 & Obligatoria & $6 \mathrm{cr}$. ECTS \\
\hline $\begin{array}{l}\text { Documentación } \\
\text { para traductores e } \\
\text { intérpretes }\end{array}$ & $2^{0}$ & Obligatoria & $6 \mathrm{cr}$. ECTS \\
\hline $\begin{array}{c}\text { Terminología para } \\
\text { traductores e } \\
\text { intérpretes }\end{array}$ & $3^{0}$ & Obligatoria & $6 \mathrm{cr}$. ECTS \\
\hline \multicolumn{4}{|c|}{ UNIVERSIDAD DE ALICANTE } \\
\hline Asignatura & Curso & Carácter & Carga lectiva \\
\hline $\begin{array}{l}\text { Lingüística aplicada } \\
\text { a la traducción }\end{array}$ & $1^{0}$ & Básica & $6 \mathrm{cr}$. ECTS \\
\hline $\begin{array}{c}\text { Terminología y } \\
\text { documentación } \\
\text { aplicadas a la } \\
\text { traducción }\end{array}$ & 10 & Básica & 6 cr. ECTS \\
\hline \multicolumn{4}{|c|}{ UNIVERSIDAD DE CÓRDOBA } \\
\hline Asignatura & Curso & Carácter & Carga lectiva \\
\hline Lengua española I & 10 & Básica & $6 \mathrm{cr}$. ECTS \\
\hline $\begin{array}{l}\text { Herramientas } \\
\text { profesionales para } \\
\text { la traducción }\end{array}$ & $2^{0}$ & Obligatoria & $6 \mathrm{cr}$. ECTS \\
\hline Terminología & $2^{0}$ & Obligatoria & $6 \mathrm{cr}$. ECTS \\
\hline $\begin{array}{c}\text { Informática aplicada } \\
\text { a la traducción }\end{array}$ & $2^{0}$ & Obligatoria & $6 \mathrm{cr}$. ECTS \\
\hline
\end{tabular}

Hikma 12 (2013), 47-63 


\begin{tabular}{|c|c|c|c|}
\hline \multicolumn{4}{|c|}{ UNIVERSIDAD DE GRANADA } \\
\hline Asignatura & Curso & Carácter & Carga lectiva \\
\hline Lengua española I & $1^{0}$ & Básica & $6 \mathrm{cr}$. ECTS \\
\hline Lengua española III & $2^{\circ}$ & Obligatoria & $6 \mathrm{cr}$. ECTS \\
\hline $\begin{array}{l}\text { El proceso de } \\
\text { traducción y sus } \\
\text { fundamentos } \\
\text { lingüísticos }\end{array}$ & $2^{\circ}$ & Básica & $6 \mathrm{cr}$. ECTS \\
\hline \multicolumn{4}{|c|}{ UNIVERSIDAD DE LAS PALMAS DE GRAN CANARIA } \\
\hline Asignatura & Curso & Carácter & Carga lectiva \\
\hline Documentación & $1 \stackrel{0}{ }$ & Básica & $6 \mathrm{cr}$. ECTS \\
\hline Terminología & $2^{\circ}$ & Básica & $6 \mathrm{cr}$. ECTS \\
\hline \multicolumn{4}{|c|}{ UNIVERSIDAD DE MÁLAGA } \\
\hline Asignatura & Curso & Carácter & Carga lectiva \\
\hline $\begin{array}{c}\text { Herramientas para } \\
\text { la práctica de la } \\
\text { traducción } \\
\text { I:Terminología }\end{array}$ & $2^{0}$ & Obligatoria & $6 \mathrm{cr}$. ECTS \\
\hline $\begin{array}{l}\text { Lengua y cultura } \\
\text { “A” aplicadas a la } \\
\text { traducción (III): } \\
\text { español }\end{array}$ & $2^{0}$ & Obligatoria & $6 \mathrm{cr}$. ECTS \\
\hline \multicolumn{4}{|c|}{ UNIVERSIDAD DE MURCIA } \\
\hline Asignatura & Curso & Carácter & Carga lectiva \\
\hline $\begin{array}{l}\text { Informática } \\
\text { aplicada a la } \\
\text { traducción II }\end{array}$ & $3^{\circ}$ & Obligatoria & $6 \mathrm{cr}$. ECTS \\
\hline Terminología & $3^{\circ}$ & Obligatoria & $6 \mathrm{cr}$. ECTS \\
\hline
\end{tabular}




\begin{tabular}{|c|c|c|c|}
\hline \multicolumn{4}{|c|}{ UNIVERSIDAD DE SALAMANCA } \\
\hline Asignatura & Curso & Carácter & Carga lectiva \\
\hline $\begin{array}{c}\text { Recursos } \\
\text { lexicográficos para } \\
\text { la traducción }\end{array}$ & $1^{0}$ & Obligatoria & $6 \mathrm{cr}$. ECTS \\
\hline $\begin{array}{l}\text { Documentación } \\
\text { para la traducción }\end{array}$ & $2^{0}$ & Básica & $6 \mathrm{cr} . \mathrm{ECTS}$ \\
\hline $\begin{array}{l}\text { Terminología } \\
\text { aplicada a la } \\
\text { traducción }\end{array}$ & $3^{0}$ & Obligatoria & $6 \mathrm{cr}$. ECTS \\
\hline $\begin{array}{c}\text { Gestión } \\
\text { terminológica y de } \\
\text { proyectos }\end{array}$ & $4^{\circ}$ & Obligatoria & $6 \mathrm{cr}$. ECTS \\
\hline \multicolumn{4}{|c|}{ UNIVERSIDAD DE VALENCIA } \\
\hline Asignatura & Curso & Carácter & Carga lectiva \\
\hline $\begin{array}{l}\text { Lengua A I: uso y } \\
\text { normativa de la } \\
\text { lengua española }\end{array}$ & $1 \stackrel{0}{ }$ & Básica & $6 \mathrm{cr}$. ECTS \\
\hline $\begin{array}{c}\text { TIC aplicada a la } \\
\text { traducción }\end{array}$ & $1^{0}$ & Obligatoria & $6 \mathrm{cr}$. ECTS \\
\hline $\begin{array}{c}\text { Terminología y } \\
\text { lexicografía }\end{array}$ & $2^{\circ}$ & Obligatoria & $6 \mathrm{cr}$. ECTS \\
\hline \multicolumn{4}{|c|}{ UNIVERSIDAD DE VIGO } \\
\hline Asignatura & Curso & Carácter & Carga lectiva \\
\hline $\begin{array}{c}\text { Lengua A II: } \\
\text { Introducción a las } \\
\text { lenguas de } \\
\text { especialización }\end{array}$ & $2^{0}$ & Obligatorio & 6 cr. ECTS \\
\hline $\begin{array}{l}\text { Herramientas para } \\
\text { la traducción y la } \\
\text { interpretación II: } \\
\text { documentación }\end{array}$ & $2^{0}$ & Obligatorio & $6 \mathrm{cr}$. ECTS \\
\hline \multicolumn{4}{|c|}{ UNIVERSIDAD DEL PAÍS VASCO } \\
\hline Asignatura & Curso & Carácter & Carga lectiva \\
\hline $\begin{array}{c}\text { Información y } \\
\text { documentación para } \\
\text { traductores e } \\
\text { intérpretes }\end{array}$ & $1^{0}=$ & Obligatorio & 6 cr. ECTS \\
\hline
\end{tabular}




\begin{tabular}{|c|c|c|c|}
\hline $\begin{array}{c}\text { Terminología de la } \\
\text { lengua } A\end{array}$ & $3^{\circ}$ & Obligatoria & $6 \mathrm{cr}$. ECTS \\
\hline \multicolumn{4}{|c|}{ UNIVERSIDAD JAUME I } \\
\hline Asignatura & Curso & Carácter & Carga lectiva \\
\hline Lengua española & $1^{\circ}$ & Básica & 6 cr. ECTS \\
\hline $\begin{array}{c}\text { Terminología para } \\
\text { traductores e } \\
\text { intérpretes }\end{array}$ & $2^{0}$ & Obligatoria & 6 cr. ECTS \\
\hline \multicolumn{4}{|c|}{ UNIVERSIDAD PABLO DE OLAVIDE } \\
\hline Asignatura & Curso & Carácter & Carga lectiva \\
\hline Terminología & 30 & Obligatorio & 3 cr. ECTS \\
\hline \multicolumn{4}{|c|}{ UNIVERSIDAD POMPEU FABRA } \\
\hline Asignatura & Curso & Carácter & Carga lectiva \\
\hline Terminología & 30 & Obligatoria & 4 cr. ECTS \\
\hline $\begin{array}{c}\text { Industrias de la } \\
\text { lengua }\end{array}$ & $3 \% / 4 \div$ & Optativa & 4 cr. ECTS \\
\hline
\end{tabular}

